\title{
Propuesta metodológica para la implementación de herramientas de gamificación en la formación de Maestros de Primaria
}

\author{
Methodological Proposal for the implementation of gamification tools in the training of \\ Primary School Teachers
}

Proposta metodològica per a la implementació d'eines de gamificació en la formació de mestres de primària

\author{
María Luisa García-Hernández ${ }^{*}$, Mónica Porto-Currás ${ }^{\circledR}$, Francisco J \\ Hernández-Valverde ${ }^{\circledR}$
}

Didáctica y Organización Escolar, Universidad de Murcia, Murcia, España

*Autor para correspondencia: luisagarcia@um.es (María Luisa García-Hernández)

Recibido: 21/04/2020 | Aceptado: 04/11/2020 | Publicado: 20/01/2021

\section{Cómo citar: García-Valverde, M.L.; Porto-Currás, M.; y Hernández-Valverde, F.J. (2021). Propuesta metodológica para la implementación de herramientas de gamificación en la formación de Maestros de Primaria. Research in Education and Learning Innovation Archives, 26,35-54. \\ 10.7203/realia.26.17137}

Copyright: El/La Autor/a. Open Access: Este es un artículo de acceso abierto distribuido bajo los términos de la licencia Creative Commons

Attribution-NoDerivatives 4.0 International licence (CC BY-ND 4.0)

Financiación: None informed
RESUMEN: Numerosas investigaciones constatan que la gamificación desarrolla escenarios favorables para el aprendizaje del alumnado, favoreciendo su implicación y fomentando un rol más activo. Desde esta premisa, en el presente artículo se muestran los resultados de una experiencia desarrollada con estudiantes de Magisterio, buscando un entorno de enseñanza que fomente su implicación personal en la construcción de aprendizajes, de forma estimulante y retadora, al mismo tiempo que les permita enfrentarse a situaciones problemáticas con responsabilidad, siendo capaces de emplear un pensamiento crítico, creativo y contextualizado, trabajando las habilidades sociales y de comunicación. Para valorar su éxito, se analizan los resultados hallados tras la implementación, comparando las respuestas del alumnado que ha seguido esta experiencia, con las respuestas de un grupo de la misma Universidad y Grado que trabajó con una metodología diferente. Como instrumento de recogida de datos se ha empleado un cuestionario validado internacionalmente, Student Engagement Questionnaire, corroborando su validez y fiabilidad con los participantes de nuestro estudio. Los resultados hallados corroboran que la experiencia metodológica basada en la gamificación ha propiciado un entorno de enseñanza-aprendizaje motivador, con alta interacción entre docentes y estudiantes, que favorece el aprendizaje autogestionado, las habilidades interpersonales, de comunicación y la adaptabilidad de los estudiantes. Se concluye que una correcta planificación y desarrollo de la gamificación permite crear entornos que enriquecen los aprendizajes en el nivel universitario, mejorando el manejo de los conocimientos y contenidos formativos y habilidades transversales de especial relevancia en la formación de futuros maestros de Educación Primaria.

PALABRAS CLAVE: metodologías; formación de profesores; ambientes de aprendizaje; motivación; gamificación; videojuegos; enseñanza

ABSTRACT: Numerous studies have confirmed that gamification develops favorable scenarios for student learning by encouraging student involvement and promoting a more active role. Starting from this premise, in this article we describe an experience we developed with first-grade teachertraining students to find a learning environment that will encourage student involvement in the construction of learning in a challenging and stimulating way. Other aims of this experience are 
to enable students to face awkward situations responsibly, work on their social and communication skills, and employ critical, creative and contextualized thinking. To evaluate the success of this experience, after its implementation we analyzed the results by comparing responses from participating students with those from a group from the same Degree and university who employed a different methodology. To gather information, we administered an internationally validated survey - the Student Engagement Questionnaire - and corroborated its validity and reliability with the participants of our study. Our main results are that this methodological experience based on gamification promoted a motivating teaching-learning environment, encouraged significant interaction between teachers and students, promoted self-managed learning, interpersonal and communicative skills, and developed student adaptability, while also creating a playful and dynamic atmosphere. We therefore conclude that proper planning and gamification development help to create environments that enrich learning at the university level, improve the management of knowledge and training content, and develop important transversal skills for the training of future Elementary School teachers.

KEYWORDS: methodology; teacher training; learning environment; motivation; gamification; video games; teaching

RESUM: Nombroses investigacions constaten el desenvolupament d'escenaris favorables per a l'aprenentatge dels alumnes que afavorisquen la seua implicació i fomenten un rol més actiu. Des d'aquesta premissa, es mostren en aquest article els resultats d'una experiència desenvolupada amb estudiants de magisteri que busquen un entorn d'ensenyament que fomente la seua implicació personal en els aprenentatges, de manera estimulant i reptadora, alhora que els permeta enfrontar-se a situacions problemàtiques amb responsabilitat i que siguen capaços d'utilitzar un pensament crític, creatiu i contextualitzat, que treballe amb les habilitats socials i de comunicació. Per valorar-ne l'èxit, s'analitzen els resultats trobats després de la posada en pràctica. Es comparen les respostes dels alumnes que han seguit aquesta experiència, amb les respostes d'un grup de la mateixa universitat i grau que va treballar amb una metodologia diferent. Com a instrument de recollida de dades, s'ha fet servir un qüestionari validat internacionalment i se n'ha corroborat la validesa i la fiabilitat amb els participants del nostre estudi. Els resultats trobats demostren que l'experiència metodològica basada en un entorn motivador, amb una alta interacció entre docents i estudiants, afavoreix l'aprenentatge, les habilitats interpersonals i de comunicació i l'adaptabilitat dels estudiants. Es conclou que una correcta planificació i desenvolupament permet crear entorns que enriqueixen els aprenentatges en el nivell universitari, milloren el maneig dels coneixements, dels continguts formatius i de les habilitats transversals d'especial rellevància en la formació de futurs mestres d'ensenyament primari.

PARAULES CLAU: metodologies; formació de professors; ambients d'aprenentatge; motivació; gamificació; videojocs; ensenyament

\section{Notas de aplicación práctica}

\section{Qué se sabe sobre este tema}

- La gamificación en el aula constituye un medio motivador para la enseñanza de diversas materias.

- La gamificación mejora la atención, la concentración, el pensamiento complejo y la planificación estratégica.

- La puesta en práctica de estrategias metodológicas innovadoras favorece un aumento en la motivación y el aprendizaje en los estudiantes.

\section{Qué añade este artículo}

- Aplicación del cuestionario SEQ validado a nivel internacional, nacional y en esta investigación

- Valoración de la experiencia de futuros maestros de educación primaria. Mejora de las competencias transversales de los futuros maestros de educación primaria a través de la gamificación. 


\section{Implicaciones para la práctica y/o política}

- Mejorar las condiciones de aprendizaje de los estudiantes universitarios a través de actividades lúdicas.

- Fomentar la coordinación entre asignaturas para trabajar estos ambientes de aprendizaje de manera interdisciplinar.

\section{INTRODUCCIÓN Y REVISIÓN DE LA LITERATURA}

La incorporación de la Universidad en el Espacio Europeo de Educación Superior (EEES) tenía como finalidad, entre otras cuestiones -de carácter menos pedagógico, potenciar un modelo de enseñanza centrado en el estudiante. Ello implica que se valore tanto el proceso de aprendizaje que se está desarrollando como el producto final, procurando que el docente ejerza más un rol de guía que uno de instructor y que el estudiante asuma en todo su proceso de aprendizaje un rol activo.

Se busca así la consecución de aprendizajes significativos y competentes, que permita a los egresados universitarios enfrentarse a situaciones problemáticas con responsabilidad, cuestionamientos éticos y siendo capaces de emplear un pensamiento crítico, creativo y contextualizado (Fernández-March, 2006; García y Álvarez Méndez, 2005).

Sin embargo, con demasiada frecuencia se leen críticas sobre la formación inicial que se ofrece en la formación de maestros de Educación Primaria (Amor-Almedina y Serrano-Rodríguez, 2019; Bozu y Arenaga, 2017; Gairín-Sallán, Díaz-Vicario, del Arco Bravo, y Alarcia, 2019; Gewerc y Alonso-Ferreiro, 2017; Medina-Moya y PérezCabrera, 2017), que provoca en los alumnos una falta de respuesta eficaz que les permita enfrentarse a las necesidades de la escuela y la sociedad actual. Si bien gran parte de estas críticas nacen de la escasa importancia de los contenidos impartidos, consideramos que no son sólo importantes los contenidos desarrollados a lo largo de su formación, sino también los métodos que se desarrollan para enseñarlos y las expectativas de los discentes sobre ellos.

La profesionalidad del docente no se reduce a la correcta selección y exposición de los contenidos en clase, sino que debe ser un profesional capaz de reflexionar a partir de su práctica y de actuar de manera eficaz en un contexto concreto, así como de innovar para adaptarse a las necesidades del alumnado. Desde esta perspectiva, debe ser un agente activo, capaz de conseguir que el alumnado desarrolle un interés por aprender, de desarrollar una metodología que facilite que puedan aprender efectivamente lo que pretenden enseñarles (Zabalza, 2007). Si además estamos hablando de alumnos del Grado de Magisterio de Educación Primaria, estas innovaciones docentes deben permitir al alumnado mejorar su capacitación profesional, darle conocimientos y recursos para poder desarrollar también estas competencias en su futuro docente.

Desde estas premisas, en este artículo se presenta los resultados obtenidos en el desarrollo de una experiencia metodológica diseñada con la pretensión de alcanzar las siguientes finalidades:

1. Mejorar las habilidades de búsqueda de información, resolución de problemas y comunicación interpersonal como competencias transversales indispensables en estudiantes universitarios.

2. Facilitar retroalimentación positiva sobre los aprendizajes que se están construyendo, de forma que permita al profesorado implicado y a los propios estudiantes conocer errores y cómo superarlos, además de los puntos fuertes que se están afianzando. 
3. Propiciar un clima de cooperación y trabajo grupal, favoreciendo la mejora de habilidades sociales fundamentales para un futuro maestro de Educación Primaria.

4. Incluir a todos los estudiantes, planteando ambientes de aprendizaje que supongan un esfuerzo y sean un reto para todos.

5. Fomentar la motivación, implicación y diversión de los estudiantes, quiénes deberán sentir que participan en un proceso de aprendizaje estimulante que les permite desarrollar aprendizajes significativos.

6. Mejorar la comprensión y personalización de contenidos complejos para alumnado de $1^{\circ}$ de Grado de Maestro en Educación Primaria.

En este contexto nos situamos el equipo docente de la asignatura Planificación de la Acción Educativa, asignatura básica del Grado de Maestros en Educación Primaria, que se imparte en el primer cuatrimestre del primer curso de su formación universitaria y que, por tanto, supone su primer acercamiento a teorías y/o concepciones de la enseñanza y aprendizaje y sobre el sentido básico que le otorgarán a su tarea docente en la primera etapa de Educación Obligatoria. Se busca plantear con los estudiantes una forma de saber hacer, saber ser y estar y conceptualizar la profesión docente y su concreción en la planificación, desarrollo y evaluación de la enseñanza, que les permita afianzar unos aprendizajes básicos que seguirán desarrollando a lo largo de los restantes cursos del Grado de formación universitaria, pero que además los implique personalmente en su construcción, de forma estimulante y retadora.

Todo ello, con la dificultad que supone tener que desarrollarlo con grupos de más de 60 alumnos y con escasas horas de trabajo presencial (enmarcados, como todos los Grados universitarios españoles, en un contexto que establece un alto número de horas de trabajo autónomo por parte del estudiantado).

Tras un proceso de documentación y búsqueda de alternativas, optamos por emplear una metodología basada en la gamificación, entendiéndola como una oportunidad para motivar, mejorar dinámicas de grupo, atención, crítica reflexiva y aprendizaje significativo de los estudiantes (Oliva, 2017). El uso del juego como herramienta de aprendizaje parece ser un enfoque prometedor debido a su habilidad para enseñar y reforzar no solo conocimientos, sino también habilidades como la resolución de problemas, la colaboración o la comunicación (Contreras y Eguía, 2016; Parra-González y Segura-Robles, 2019). Se considera así que mediante los juegos es posible el desarrollo de habilidades sociales (Perrotta, Featherstone, Aston, y Houghton, 2013), la motivación hacia el aprendizaje (Kenny y McDaniel, 2011; López-Belmonte, Segura-Robles, Fuentes-Cabrera, y Parra-González, 2020; Parra-González y Segura-Robles, 2019) una mejora en la atención, la concentración, el pensamiento complejo y la planificación estratégica (Kirriemuir y Mcfarlane, 2004). Incluso ayudan a interiorizar conocimientos multidisciplinarios (Mitchell y Savill-Smith, 2004) propician un pensamiento lógico y crítico y a mejorar habilidades que ayudan a resolver diversos problemas (Contreras y Eguía, 2016; Higgins, Grant, y Shah, 1999).

Finalmente, podemos resaltar que la gamificación en el aula constituye un medio poderoso para la enseñanza- y el aprendizaje- de diversas materias, fomenta el trabajo en equipo y favorece la sociabilidad del alumno, además de mejorar procesos cognitivos y promover el compromiso y la motivación (Montes-Rodríguez, 2018).

Para la implementación de esta experiencia de gamificación se optó por la herramienta Kahoot. Se trata de una aplicación que permite elaborar preguntas o cuestionarios para que los alumnos respondan, a través de sus teléfonos móviles, tabletas u ordenadores portátiles, a las cuestiones planteadas por el docente sobre los contenidos de la asignatura. Además, permite desarrollar las competencias transversales de la Titulación, utilizar las TIC como recurso de aprendizaje, aumentar la motivación de los estudiantes y obtener información del proceso de aprendizaje y comprensión 
de los contenidos por parte de los estudiantes. Por otra parte, contribuye a mejorar la participación e implicación de los estudiantes en el aula y fomenta las relaciones positivas entre los grupos; siendo una potencial herramienta de gamificación en el aprendizaje (Díaz Barriga, 2013 (Cerro, 2015; Fuertes et al., 2016; Moya et al., 2016; Parra-González y Segura-Robles, 2019).

Los aspectos comentados se ven plasmados en otras experiencias (Pintor-Díaz, 2017), donde los resultados de su trabajo muestran como el alumnado considera que trabajar con la herramienta kahoot en el aula generó, entre otros aspectos, un aprendizaje más activo, una mayor motivación y un aprendizaje significativo basado en la relación entre la teoría y la práctica y en la retroalimentación dada entre docente y alumnado.

También las conclusiones establecidas (Guzmán-Duque, Mendoza-Paredes, y Tavera-Castillo, 2018; López-Belmonte et al., 2020), corroboran que la gamificación desarrolla escenarios propicios para el aprendizaje del alumnado, ya que fomenta su implicación, provoca un rol activo del estudiante y favorece su habilidad para incorporar las tecnologías a los procesos de enseñanza-aprendizaje.

\section{MATERIALES Y MÉTODOS}

Este artículo tiene por objetivo exponer los resultados de una experiencia metodológica que busca mejorar la capacidad de autorregulación del aprendizaje (por parte del alumnado) a través de un ambiente de enseñanza-aprendizaje motivador, dinámico y desafiante.

Para valorar su desarrollo y resultados, se plantea un diseño no experimental de finalidad descriptiva. Los estudios de corte descriptivo pretenden "describir fenómenos, situaciones, contextos y sucesos" (Sampieri, Collado, y Lucio, 2014), con la finalidad de conocer cómo son y cómo se manifiestan. Concretamente, estas investigaciones aspiran a conocer las características del grupo, del proceso o del suceso que se estudia.

El estudio se desarrolla en el marco institucional de la Universidad de Murcia y la Facultad de Educación, focalizado en el Grado de Maestro en Educación Primaria.

La población de referencia la conforman un total de 428 alumnos matriculados en la asignatura Planificación de la Acción Educativa ( $1^{\circ}$ curso Grado Maestro Educación Primaria) en el curso 2018-2019.

Esta población se distribuye en 7 grupos: 4 con horario de mañana y los 3 restantes, horario vespertino. Además, uno de los grupos con horario matinal forma parte de un programa de estudios Bilingüe, por lo que utiliza materiales y metodologías diferentes a los grupos restantes.

La muestra productora de datos la conforman 233 estudiantes, distribuidos entre los tres grupos de mañana que no forman parte del Plan Bilingüe y un grupo de control con alumnos del horario de tarde, tal y como recoge la Tabla 1.

Tabla 1. Distribución de la muestra. Fuente: elaboración propia

\begin{tabular}{lccc}
\hline Grupos & Población & Muestra productora de datos & \% de participación \\
\hline Mañana no bilingüe & 208 & 177 & $85,09 \%$ \\
Tarde & 162 & 56 & $34,56 \%$ \\
\hline
\end{tabular}

Desde un enfoque metodológico cuantitativo, el análisis atiende a la visión que manifiestan los estudiantes sobre el entorno de E-A creado y las capacidades de aprendizaje que han podido desarrollar.

Para conocer el grado de cumplimiento percibido por cada alumno se le ofrece un cuestionario validado a nivel internacional: el cuestionario SEQ (Student Engagement Questionnaire, Kember y Leung, 2009).

Se selecciona este cuestionario dado que nos interesa recoger información de las capacidades desarrolladas por los estudiantes y las percepciones que estos alumnos 
tienen sobre la capacidad del profesorado para articular un entorno rico y constructivo para el aprendizaje. Con relación a ello, este instrumento permite realizar un análisis riguroso de las fortalezas y debilidades de estas dimensiones, que quedan configuradas en el cuestionario de la siguiente manera: variables del entorno de enseñanzaaprendizaje y variables de las capacidades del alumnado.

Concretamente, el cuestionario SEQ consta de 35 ítems y delimita siete capacidades del alumno (Pensamiento crítico; Pensamiento creativo; Aprendizaje autogestionado; Adaptabilidad; Resolución de problemas; Habilidades interpersonales; Habilidades de comunicación) y ocho del entorno de enseñanza-aprendizaje (Aprendizaje activo; Enseñanza para la comprensión; Evaluación; Coherencia del currículum; Interacción profesor-estudiante; Feedback para ayudar al aprendizaje; Relación con otros estudiantes; Aprendizaje cooperativo), aplicadas a través de una escala tipo Likert con cuatro opciones de respuesta: 1 (muy en desacuerdo), 2 (desacuerdo), 3 (de acuerdo) y 4 (muy de acuerdo).

A esta información, nos interesaba sumarle la satisfacción que los estudiantes tenían con la experiencia. Es por ello por lo que, al final, se incluyen tres preguntas específicas que permiten valorar esta experiencia con la herramienta Kahoot (ver Tabla 2).

Tabla 2. Preguntas sobre la herramienta Kahoot. Fuente: elaboración propia

36. Considero que la plataforma Kahoot es adecuada para mejorar los aprendizajes en el nive universitario.

37. Después de contestar las preguntas formuladas en la plataforma Kahoot, he logrado un mayor manejo de los conocimientos y contenidos de la asignatura.

38. Estimo que la herramienta Kahoot es un recurso que permite ir mejor preparado para el momento de realizar el examen

La experiencia metodológica se lleva a cabo en 4 fases (sintetizadas en la Tabla 3). En la primera de ellas se diseña la experiencia, delimitando la asignatura, los grupos participantes, el recurso a utilizar para la implementación de la gamificación y la recompensa a obtener por los estudiantes, que en este caso supone la consecución de 2 puntos en la evaluación final de la asignatura (sobre 10), mientras que los demás deben obtener esos puntos en el examen final.

Lo que se pretende con esto es un aprendizaje de contenidos más profundo, que sean capaces de comprehender contenidos que son más complicados (siendo alumnos de $1^{\circ}$ curso) de una manera más lúdica y, evidentemente, que ese aprendizaje y experiencia tenga una repercusión en los resultados académicos.

Tabla 3. Fases del desarrollo de la experiencia. Fuente: elaboración propia

\begin{tabular}{|c|c|c|}
\hline Fase & Desarrollo & Resultado \\
\hline Fase I & $\begin{array}{l}\text { Planificación y diseño de la } \\
\text { experiencia }\end{array}$ & $\begin{array}{l}\text { Se concretaron las asignaturas en las que se } \\
\text { realizaría la experiencia, el recurso utilizado para } \\
\text { implementar la gamificación y las recompensas }\end{array}$ \\
\hline Fase II & $\begin{array}{l}\text { Diseño de los Kahoot para cada } \\
\text { tema o bloque de contenidos }\end{array}$ & $\begin{array}{l}\text { Se diseñaron los diversos Kahoot para cada una } \\
\text { de las materias considerando cuestiones } \\
\text { planteadas por los estudiantes durante el } \\
\text { desarrollo del tema o bloque de contenidos. }\end{array}$ \\
\hline Fase III & $\begin{array}{l}\text { Implementación de la experiencia } \\
\text { de gamificación en el aula }\end{array}$ & $\begin{array}{l}\text { Se desarrolló la metodología de gamificación en } \\
\text { el aula. }\end{array}$ \\
\hline Fase IV & $\begin{array}{l}\text { Valoración de la experiencia por } \\
\text { parte del alumnado }\end{array}$ & $\begin{array}{l}\text { Se administró el cuestionario SEQ (Student } \\
\text { Engagement Questionnaire), ampliado, para } \\
\text { conocer la valoración de la experiencia por parte } \\
\text { del alumnado. }\end{array}$ \\
\hline
\end{tabular}


En la segunda fase se realiza el diseño de los diversos test Kahoot. Al trabajar con grupos de alumnos numerosos (aproximadamente 60) se decide desarrollar los test kahoot grupalmente, organizando los alumnos en equipos de $4-5$ personas.

Cada docente negocia con el alumnado cuándo y cómo realizar los test kahoot, al final de cada tema o después de cada bloque de contenidos. Asimismo, se opta por que los estudiantes elaboren preguntas para que sean tenidas en cuenta por el profesorado en la confección de cada kahoot, lo que permite a los alumnos estudiar y afianzar los contenidos de la materia, combinándolas con las diseñadas por el propio docente. Se busca así que los estudiantes optimicen el estudio del tema o bloque con la búsqueda de posibles preguntas.

En la fase III se implementa la experiencia en el aula de tal manera que, al finalizar cada tema o bloque, el docente proyecta las cuestiones referidas al mismo y los estudiantes, agrupados en equipos, a través de sus Smartphone, y ajustándose el tiempo planteado por cada docente, deben seleccionar la respuesta correcta entre las opciones planteadas.

Cabe destacar que este proceso permite, por un lado, evaluar en el mismo momento los conocimientos aprendidos por los estudiantes, ya que la herramienta ofrece información sobre los grupos que responden correctamente y la rapidez con que lo hacen; y, por otra parte, solucionar las dudas que pueden surgir respecto a determinadas cuestiones planteadas o sobre las respuestas correctas o incorrectas, a través del feedback que el profesorado ofrece mientras se desarrollan los test kahoot.

De esta manera, el grupo que obtiene mayor puntuación en cada uno de los kahoot desarrollados accede a participar en un kahoot final, con cuestiones referentes a todos los temas o bloques de contenidos de la asignatura. Los miembros del grupo ganador de este último kahoot obtienen la gamificación citada anteriormente.

Por último, en la fase IV, se pide a los estudiantes que cumplimenten el cuestionario SEQ ampliado, a fin de conocer su valoración sobre la experiencia llevada a cabo.

\section{ANÁLISIS Y RESULTADOS}

Los datos se analizaron con el programa estadístico SPSS v24.

Para analizar la estructura interna del SEQ se realiza un análisis factorial exploratorio de ejes principales con rotación varimax. Posteriormente, se evalúa el ajuste del modelo planteado. Un buen ajuste de los datos al modelo viene dado por el estadístico $\chi^{2}$, que debe ser pequeño y no significativo.

Los datos proporcionados (Gargallo et al., 2018 FALTA REFERENCIA), demuestran que la estructura dimensional planteada en el SEQ se sostiene con los datos recogidos en una muestra española. La validación del cuestionario con la muestra de nuestra investigación corrobora esta conclusión, al comprobar que la estructura de dimensiones del modelo y de sus relaciones se sostiene convenientemente a partir de los resultados obtenidos mediante el chi-cuadrado, puesto que la significación es inferior a $0,05(0,000)$.

Por otro lado, se obtiene información sobre el coeficiente alfa de Cronbach, siguiendo el protocolo de validación del SEQ (Gargallo et al., 2018 FALTA REFERENCIA). En este sentido, resaltar el alto grado de correlación entre los elementos (a mayor valor de Alfa mayor fiabilidad) siendo el valor del alfa de Cronbach con nuestra muestra de 0,948 .

Finalmente, las respuestas del cuestionario se analizan mediante:

- Estadísticos descriptivos, englobando la media, mediana y moda (medidas de tendencia central), organizados en función de las dimensiones y variables del cuestionario y atendiendo a la distribución del alumnado en los grupos que siguieron la experiencia y el grupo de tarde. 
- Pruebas de normalidad: prueba de Kolmogorov-Smirnov, por tratarse de una muestra mayor a 50. Los resultados de esta prueba indican que la distribución de los datos no cumple los supuestos de normalidad ( $p=, 000$, siendo esta menor a 0,05 ) (ver anexo 1).

- U de Mann-Whitney, prueba no paramétrica que permite comparar dos muestras independientes cuando no son asumibles los supuestos de normalidad, con la finalidad de determinar las posibles diferencias entre las valoraciones de los estudiantes de los grupos de mañana y del grupo de tarde.

\subsection{Bloque 1. Capacidades}

A continuación, analizamos las respuestas del alumnado a las cuestiones de este bloque diferenciando entre el grupo de estudiantes participantes en la experiencia metodológica de mañana (grupo A) y el grupo que siguió una metodología diferente, grupo de tarde (grupo B). En la Tabla 4 se muestran los descriptivos de las valoraciones que realiza el grupo A referentes a estas cuestiones.

Tabla 4. Respuestas del grupo A, a los ítems relacionados con las capacidades del alumno. Fuente: elaboración propia.

\begin{tabular}{|c|c|c|c|c|c|}
\hline & $\mathrm{N}$ & Mínimo & Máximo & Media & $\begin{array}{l}\text { Desviación } \\
\text { estándar }\end{array}$ \\
\hline $\begin{array}{l}\text { 1. He desarrollado mi capacidad } \\
\text { de enjuiciar puntos de vista } \\
\text { alternativos }\end{array}$ & 177 & 2 & 4 & 3,29 &, 566 \\
\hline $\begin{array}{l}\text { 2. Me he vuelto más dispuesto a } \\
\text { considerar diferentes puntos de } \\
\text { vista }\end{array}$ & 177 & 2 & 4 & 3,40 &, 557 \\
\hline $\begin{array}{l}\text { 3. He sido animado a usar mi } \\
\text { propia iniciativa }\end{array}$ & 177 & 2 & 4 & 3,48 &, 585 \\
\hline $\begin{array}{l}\text { 4. He sido desafiado a llegar a } \\
\text { nuevas ideas, }\end{array}$ & 176 & 1 & 4 & 3,35 & ,632 \\
\hline $\begin{array}{l}\text { 5. Siento que puedo asumir la } \\
\text { responsabilidad de mi propio } \\
\text { aprendizaje, }\end{array}$ & 176 & 1 & 4 & 3,39 & ,622 \\
\hline $\begin{array}{l}\text { 6. He conseguido mayor confianza } \\
\text { en mi habilidad para continuar } \\
\text { aprendiendo, }\end{array}$ & 177 & 1 & 4 & 3,27 & ,703 \\
\hline $\begin{array}{l}\text { 7. En esta asignatura he } \\
\text { aprendido a ser más flexible, }\end{array}$ & 177 & 1 & 4 & 3,28 & ,639 \\
\hline $\begin{array}{l}\text { 8. Me he vuelto más dispuesto a } \\
\text { cambiar mis puntos de vista y } \\
\text { aceptar nuevas ideas, }\end{array}$ & 176 & 2 & 4 & 3,45 &, 564 \\
\hline $\begin{array}{l}\text { 9. He mejorado mi capacidad de } \\
\text { utilizar el conocimiento para } \\
\text { resolver problemas en mi campo } \\
\text { de estudio, }\end{array}$ & 177 & 1 & 4 & 3,36 & ,625 \\
\hline $\begin{array}{l}\text { 10. Soy capaz de aportar } \\
\text { información y diferentes ideas } \\
\text { para resolver problemas, }\end{array}$ & 177 & 2 & 4 & 3,44 &, 562 \\
\hline $\begin{array}{l}\text { 11. He desarrollado mi capacidad } \\
\text { de comunicarme eficazmente con } \\
\text { los demás, }\end{array}$ & 177 & 1 & 4 & 3,38 & ,629 \\
\hline $\begin{array}{l}\text { 12. En esta materia he mejorado } \\
\text { mi capacidad para transmitir ideas, }\end{array}$ & 175 & 2 & 4 & 3,29 & ,615 \\
\hline
\end{tabular}




\begin{tabular}{lccccc}
\hline \multicolumn{1}{c}{ Table 4 continued } & & & & & \\
\hline $\begin{array}{l}\text { 13. He aprendido a ser un } \\
\text { miembro efectivo para el trabajo } \\
\text { en grupo, }\end{array}$ & 175 & 1 & 4 & 3,51 &, 664 \\
$\begin{array}{l}\text { 14. Me siento seguro en el trato } \\
\text { con una amplia gama de personas, }\end{array}$ & 175 & 1 & 4 & 3,42 &, 655 \\
$\begin{array}{l}\text { 15. Me siento seguro usando } \\
\text { aplicaciones informáticas cuando } \\
\text { sea necesario, }\end{array}$ & 177 & 1 & 4 & 3,44 &, 715 \\
$\begin{array}{l}\text { 16. He aprendido más sobre el } \\
\text { uso de ordenadores para } \\
\text { presentar la información, }\end{array}$ & 177 & 1 & 4 & 3,13 & \\
$\mathrm{~N}$ válido (por lista) & 169 & & & & \\
\hline
\end{tabular}

Tabla 5. Respuestas del grupo A, a los ítems relacionados con las capacidades del alumno. Fuente: elaboración propia.

\begin{tabular}{|c|c|c|c|c|c|c|c|c|c|c|c|c|c|c|c|c|}
\hline & 1 & 2 & 3 & 4 & 5 & 6 & 7 & 8 & 9 & 10 & 11 & 12 & 13 & 14 & 15 & 16 \\
\hline Válido & 177 & 177 & 177 & 176 & 176 & 177 & 177 & 176 & 177 & 177 & 177 & 175 & 175 & 175 & 177 & 177 \\
\hline Perdidos & 0 & 0 & 0 & 1 & 1 & 0 & 0 & 1 & 0 & 0 & 0 & 2 & 2 & 2 & 0 & 0 \\
\hline Media & 3,29 & 3,40 & 3,48 & 3,35 & 3,39 & 3,27 & 3,28 & 3,45 & 3,36 & 3,44 & 3,38 & 3,29 & 3,51 & 3,42 & 3,44 & 3,13 \\
\hline Mediana & 3,00 & 3,00 & 4,00 & 3,00 & 3,00 & 3,00 & 3,00 & 3,00 & 3,00 & 3,00 & 3,00 & 3,00 & 4,00 & 4,00 & 4,00 & 3,00 \\
\hline Moda & 3 & 3 & 4 & 3 & 3 & 3 & 3 & 4 & 3 & 3 & 3 & 3 & 4 & 4 & 4 & 3 \\
\hline
\end{tabular}

Tabla 6. Respuestas del grupo B a los ítems relacionados con las capacidades del alumnado. Fuente: elaboración propia

\begin{tabular}{|c|c|c|c|c|c|}
\hline & N & Mínimo & Máximo & Media & $\begin{array}{l}\text { Desviación } \\
\text { estándar }\end{array}$ \\
\hline $\begin{array}{l}\text { 1. He desarrollado mi capacidad para } \\
\text { enjuiciar puntos de vista alternativos. }\end{array}$ & 56 & 1 & 4 & 2,88 & ,541 \\
\hline $\begin{array}{l}\text { 2. Me he vuelto más dispuesto a } \\
\text { considerar diferentes puntos de vista. }\end{array}$ & 56 & 1 & 4 & 2,96 & ,785 \\
\hline $\begin{array}{l}\text { 3. He sido animado a usar mi propia } \\
\text { iniciativa. }\end{array}$ & 56 & 1 & 4 & 2,91 & ,769 \\
\hline $\begin{array}{l}\text { 4. He sido desafiado a llegar a nuevas } \\
\text { ideas. }\end{array}$ & 56 & 1 & 4 & 2,91 & ,880 \\
\hline $\begin{array}{l}\text { 5. Siento que puedo asumir la } \\
\text { responsabilidad de mi propio } \\
\text { aprendizaje. }\end{array}$ & 56 & 1 & 4 & 3,00 & ,809 \\
\hline $\begin{array}{l}\text { 6. He conseguido mayor confianza en } \\
\text { mi habilidad para continuar } \\
\text { aprendiendo. }\end{array}$ & 56 & 1 & 4 & 2,89 & ,846 \\
\hline $\begin{array}{l}\text { 7. En esta asignatura he aprendido a } \\
\text { ser más flexible. }\end{array}$ & 56 & 1 & 4 & 2,59 & ,733 \\
\hline $\begin{array}{l}\text { 8. Me he vuelto más dispuesto a } \\
\text { cambiar mis puntos de vista y aceptar } \\
\text { nuevas ideas. }\end{array}$ & 56 & 1 & 4 & 2,84 & ,757 \\
\hline $\begin{array}{l}\text { 9. He mejorado mi capacidad de } \\
\text { utilizar el conocimiento para resolver } \\
\text { problemas en mi campo de estudio. }\end{array}$ & 56 & 1 & 4 & 2,91 & ,745 \\
\hline
\end{tabular}




\begin{tabular}{|c|c|c|c|c|c|}
\hline \multicolumn{6}{|l|}{ Table 6 continued } \\
\hline & $\mathrm{N}$ & Mínimo & Máximo & Media & $\begin{array}{l}\text { Desviación } \\
\text { estándar }\end{array}$ \\
\hline $\begin{array}{l}\text { 10. Soy capaz de aportar información y } \\
\text { diferentes ideas para resolver } \\
\text { problemas. }\end{array}$ & 56 & 1 & 4 & 3,02 & ,751 \\
\hline $\begin{array}{l}\text { 11. He desarrollado mi capacidad de } \\
\text { comunicarme eficazmente con los } \\
\text { demás. }\end{array}$ & 56 & 1 & 4 & 2,95 & 796 \\
\hline $\begin{array}{l}\text { 12. En esta materia he mejorado } \mathrm{mi} \\
\text { capacidad para transmitir ideas. }\end{array}$ & 56 & 1 & 4 & 2,64 & ,724 \\
\hline $\begin{array}{l}\text { 13. He aprendido a ser un miembro } \\
\text { efectivo para el trabajo en grupo. }\end{array}$ & 56 & 1 & 4 & 3,11 & ,802 \\
\hline $\begin{array}{l}\text { 14. Me siento seguro en el trato con } \\
\text { una amplia gama de personas. }\end{array}$ & 56 & 1 & 4 & 2,77 & 809 \\
\hline $\begin{array}{l}\text { 15. Me siento seguro usando } \\
\text { aplicaciones informáticas cuando sea } \\
\text { necesario. }\end{array}$ & 56 & 1 & 4 & 2,88 & ,896 \\
\hline $\begin{array}{l}\text { 16. He aprendido más sobre el uso de } \\
\text { ordenadores para presentar la } \\
\text { información. }\end{array}$ & 56 & 1 & 4 & 2,29 & 948 \\
\hline N válido (por lista) & 56 & & & & \\
\hline
\end{tabular}

De acuerdo con los datos observados en la Tabla 4, la media obtenida en los distintos ítems evidencia que el alumnado está de acuerdo con que la utilización de esta metodología favorece el desarrollo de determinadas capacidades. Los alumnos han valorado de forma muy positiva aspectos como que esta experiencia les ha permitido aprender a ser un miembro efectivo para el grupo de trabajo $(3,51)$, han tenido una mayor iniciativa $(3,48)$ y han sido capaces de aceptar nuevas ideas aportadas por los demás. Observamos también como los ítems 16 (referido al aprendizaje sobre el uso de ordenadores) y el ítem 6 (su habilidad para continuar aprendiendo) son los que presentan un valor medio más reducido (3,13 y 3,27 respectivamente) aunque con la desviación más alta, lo que refleja discrepancias entre el alumnado.

Respecto a la moda, en la Tabla 5 se observa como en los 16 ítems que conforman la dimensión capacidades del alumno, se sitúa entre el valor 3 (de acuerdo) y el valor 4 (muy de acuerdo). Así pues, valoran de manera muy positiva la experiencia de gamificación vivida en el aula universitaria.

En contraste a lo indicado, como observamos en la Tabla 6, la media de los ítems alcanzada en el grupo B, en relación a la dimensión capacidades del alumnado, difiere de la obtenida en el grupo A.

Se observa como únicamente en tres de los 16 ítems la media se sitúa en el valor 3 (de acuerdo), siendo el valor medio más alto el relativo al aprendizaje efectivo para el trabajo en grupo (ítem 13), un valor máximo que no llega al valor medio más bajo en el grupo A. Estas diferencias se pueden observar en la Tabla 7 al analizar los resultados de los dos grupos a través de la prueba U de Mann-Whitney.

En ella se puede constatar una diferencia significativa en todos los ítems al encontrarse el nivel de significación por debajo de 0,05.

Efectivamente, discutir las ideas o el material de la asignatura con otros estudiantes fuera de las clases de Planificación de la Acción Educativa parece ser lo único que no se ha fomentado con esta experiencia metodológica, aunque la desviación de las respuestas dadas a esas cuestiones es elevada. Así, se puede observar como el valor de media más bajo es el que corresponde al ítem 32, pero también es el que se encuentra con una desviación estándar más alto, lo cual implica discrepancias entre alumnos que sí han discutido ideas fuera de la clase y alumnos que sólo tenían esta interacción en 


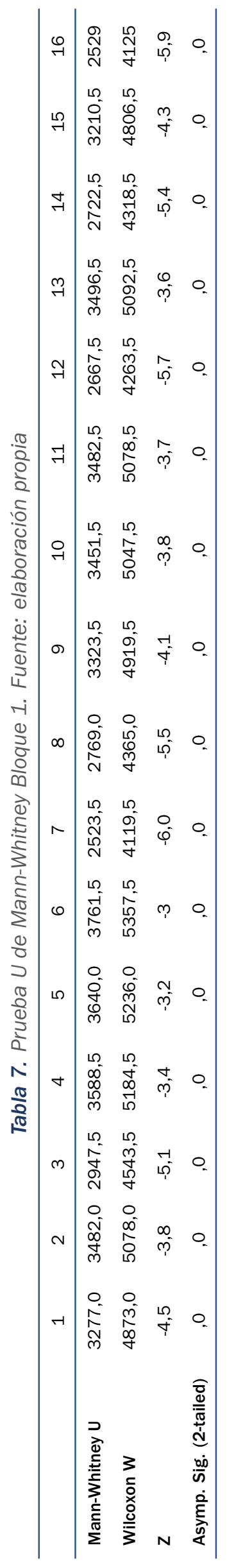


Tabla 8. Respuestas del grupo A a los ítems relacionados con el entorno de enseñanza-aprendizaje. Fuente: elaboración propia

\begin{tabular}{|c|c|c|c|c|c|}
\hline & $\mathrm{N}$ & Mínimo & Máximo & Media & $\begin{array}{l}\text { Desviación } \\
\text { estándar }\end{array}$ \\
\hline $\begin{array}{l}\text { 17. El profesor utiliza variedad de } \\
\text { métodos de enseñanza. }\end{array}$ & 177 & 1 & 4 & 3,46 & 691 \\
\hline $\begin{array}{l}\text { 18. A los estudiantes se les da la } \\
\text { oportunidad de participar en las clases. }\end{array}$ & 177 & 2 & 4 & 3,71 & ,469 \\
\hline $\begin{array}{l}\text { 19. El profesor se esfuerza por ayudar a } \\
\text { entender el material de la asignatura. }\end{array}$ & 176 & 1 & 4 & 3,61 & ,595 \\
\hline $\begin{array}{l}\text { 20. El diseño de la asignatura ayuda a } \\
\text { los estudiantes a entender sus } \\
\text { contenidos. }\end{array}$ & 176 & 1 & 4 & 3,28 & ,812 \\
\hline $\begin{array}{l}\text { 21. Cuando tengo dificultades con los } \\
\text { materiales de aprendizaje, las } \\
\text { explicaciones dadas por el profesor son } \\
\text { útiles. }\end{array}$ & 175 & 1 & 4 & 3,45 & 692 \\
\hline $\begin{array}{l}\text { 22. Hay suficiente feedback sobre las } \\
\text { actividades y tareas para asegurarse de } \\
\text { que aprendemos del trabajo que } \\
\text { hacemos. }\end{array}$ & 176 & 1 & 4 & 3,40 & 634 \\
\hline $\begin{array}{l}\text { 23. En la asignatura se utiliza variedad } \\
\text { de métodos de evaluación. }\end{array}$ & 176 & 1 & 4 & 3,40 & 616 \\
\hline $\begin{array}{l}\text { 24. Para hacerlo bien al ser evaluado en } \\
\text { esta materia necesitas tener una buena } \\
\text { capacidad de análisis. }\end{array}$ & 175 & 2 & 4 & 3,37 & 601 \\
\hline $\begin{array}{l}\text { 25. La evaluación valora nuestra } \\
\text { comprensión de los conceptos clave en } \\
\text { esta materia. }\end{array}$ & 174 & 2 & 4 & 3,42 & ,571 \\
\hline $\begin{array}{l}\text { 26. La comunicación entre el profesor y } \\
\text { los estudiantes es buena. }\end{array}$ & 177 & 2 & 4 & 3,67 & ,528 \\
\hline $\begin{array}{l}\text { 27. El profesor ayuda cuando se le } \\
\text { pregunta. }\end{array}$ & 177 & 2 & 4 & 3,73 & ,494 \\
\hline $\begin{array}{l}\text { 28. Me las arreglo para completar los } \\
\text { requisitos del programa sin sentirme } \\
\text { excesivamente estresado. }\end{array}$ & 176 & 1 & 4 & 3,05 & ,820 \\
\hline $\begin{array}{l}\text { 29. La cantidad de trabajo que se nos } \\
\text { pide que hagamos es bastante } \\
\text { razonable. }\end{array}$ & 176 & 1 & 4 & 3,08 & 871 \\
\hline $\begin{array}{l}\text { 30. Tengo un fuerte sentido de } \\
\text { pertenencia a mi grupo de clase. }\end{array}$ & 176 & 1 & 4 & 3,34 & 690 \\
\hline $\begin{array}{l}\text { 31. Con frecuencia trabajo con los } \\
\text { compañeros en mis clases. }\end{array}$ & 177 & 1 & 4 & 3,53 & 613 \\
\hline $\begin{array}{l}\text { 32. He discutido frecuentemente ideas } \\
\text { de la asignatura con otros estudiantes } \\
\text { fuera de clase. }\end{array}$ & 176 & 1 & 4 & 2,61 & ,950 \\
\hline $\begin{array}{l}\text { 33. Discutir el material de la asignatura } \\
\text { con otros estudiantes fuera de las } \\
\text { clases me ha ayudado a lograr una mejor } \\
\text { comprensión de la materia. }\end{array}$ & 176 & 1 & 4 & 2,78 & ,883 \\
\hline $\begin{array}{l}\text { 34. Puedo ver cómo encajan las } \\
\text { materias para hacer un programa de } \\
\text { estudio coherente para mi especialidad. }\end{array}$ & 175 & 1 & 4 & 3,17 & ,712 \\
\hline $\begin{array}{l}\text { 35. El programa de estudios de mi } \\
\text { especialidad está bien integrado. }\end{array}$ & 176 & 1 & 4 & 3,20 & 668 \\
\hline
\end{tabular}




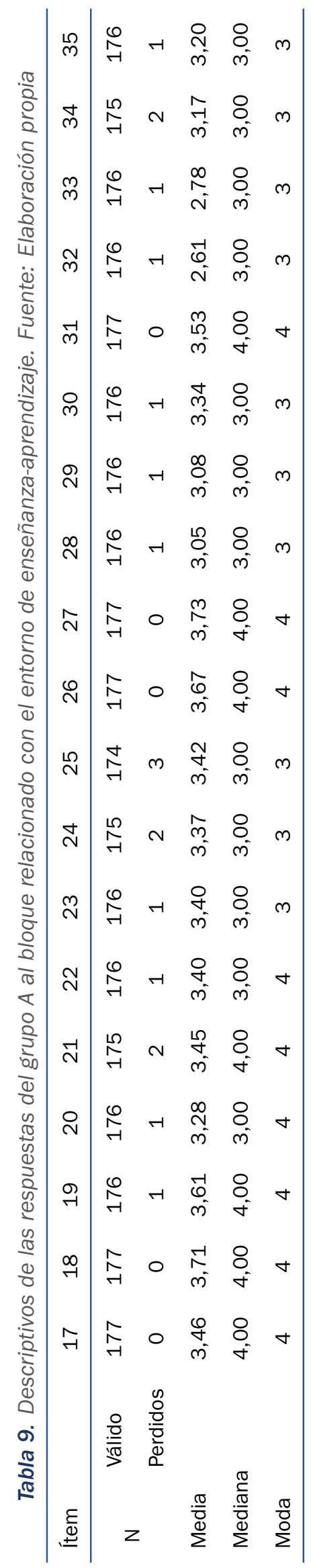


las clases.

Por el contrario, los valores más altos de media (superior a 3,5 sobre 4) se corresponden con los ítems que hacen referencia a la ayuda de los docentes o al fomento de la participación en clase, es decir, aquellos ítems que se relacionan con las variables de interacción entre profesor y estudiante (ítems 26 y 27), fomento de aprendizaje activo (ítem 18), enseñanza para la comprensión (ítem 19) o relación entre estudiantes (ítem 31). Estos datos se corroboran con el análisis de la mediana y la moda, como se puede observar en laTabla 9 .

$\mathrm{Al}$ igual que en el bloque anterior, a continuación, analizamos las respuestas del alumnado a estas cuestiones diferenciando entre el grupo de estudiantes que cursó esta asignatura en horario de mañana (grupo A) y, por tanto, siguió la experiencia metodológica antes descrita y el grupo de estudiantes del grupo de tarde que siguió una metodología diferente (grupo B). En primer lugar, se muestra una relación de los descriptivos que resumen las respuestas del alumnado del grupo A (Tabla 8).

De acuerdo con los datos que arroja la Tabla 8, la media de las respuestas dadas por el grupo A muestra que en todos los ítems los estudiantes manifiestan su acuerdo claro (en muchos casos rozando el muy de acuerdo), salvo en los ítems que se refieren a la interacción con otros estudiantes fuera de las clases.

Tal y como se puede comprobar en la $\$$, la respuesta a ningún ítem alcanza el valor medio 3 (de acuerdo), a excepción del de trabajar con los compañeros en clase, lo cual indica que los estudiantes de este grupo no están de acuerdo en que se haya conseguido un entorno de enseñanza-aprendizaje favorecedor de aprendizajes activos y significativos.

Tabla 10. Respuestas del grupo B a los ítems relacionados con el entorno de enseñanza-aprendizaje. Fuente: elaboración propia

\begin{tabular}{|c|c|c|c|c|c|}
\hline & $\mathrm{N}$ & Mínimo & Máximo & Media & $\begin{array}{l}\text { Desviación } \\
\text { estándar }\end{array}$ \\
\hline $\begin{array}{l}\text { 17. El profesor utiliza variedad de } \\
\text { métodos de enseñanza. }\end{array}$ & 56 & 1 & 4 & 2,34 & ,815 \\
\hline $\begin{array}{l}\text { 18. A los estudiantes se les da la } \\
\text { oportunidad de participar en las } \\
\text { clases. }\end{array}$ & 56 & 1 & 4 & 2,63 & ,822 \\
\hline $\begin{array}{l}\text { 19. El profesor se esfuerza por } \\
\text { ayudar a entender el material de la } \\
\text { asignatura. }\end{array}$ & 56 & 1 & 4 & 2,66 & ,880 \\
\hline $\begin{array}{l}\text { 20. El diseño de la asignatura } \\
\text { ayuda a los estudiantes a entender } \\
\text { sus contenidos. }\end{array}$ & 56 & 1 & 4 & 2,57 & ,759 \\
\hline $\begin{array}{l}\text { 21. Cuando tengo dificultades con } \\
\text { los materiales de aprendizaje, las } \\
\text { explicaciones dadas por el profesor } \\
\text { son útiles. }\end{array}$ & 56 & 1 & 4 & 2,61 & ,846 \\
\hline $\begin{array}{l}\text { 22. Hay suficiente feedback sobre } \\
\text { las actividades y tareas para } \\
\text { asegurarse de que aprendemos del } \\
\text { trabajo que hacemos. }\end{array}$ & 56 & 1 & 4 & 2,46 & ,785 \\
\hline $\begin{array}{l}\text { 23. En la asignatura se utiliza } \\
\text { variedad de métodos de evaluación. }\end{array}$ & 56 & 1 & 4 & 2,43 & ,684 \\
\hline $\begin{array}{l}\text { 24. Para hacerlo bien al ser } \\
\text { evaluado en esta materia necesitas } \\
\text { tener una buena capacidad de } \\
\text { análisis. }\end{array}$ & 56 & 1 & 4 & 2,73 & ,674 \\
\hline
\end{tabular}




\begin{tabular}{|c|c|c|c|c|c|}
\hline \multicolumn{6}{|l|}{ Table 10 continued } \\
\hline & N & Mínimo & Máximo & Media & $\begin{array}{l}\text { Desviación } \\
\text { estándar }\end{array}$ \\
\hline $\begin{array}{l}\text { 25. La evaluación valora nuestra } \\
\text { comprensión de los conceptos } \\
\text { clave en esta materia. }\end{array}$ & 56 & 1 & 4 & 2,77 & ,660 \\
\hline $\begin{array}{l}\text { 26. La comunicación entre el } \\
\text { profesor y los estudiantes es } \\
\text { buena. }\end{array}$ & 56 & 1 & 4 & 2,50 & ,874 \\
\hline $\begin{array}{l}\text { 27. El profesor ayuda cuando se le } \\
\text { pregunta. }\end{array}$ & 56 & 1 & 4 & 2,86 & ,796 \\
\hline $\begin{array}{l}\text { 28. Me las arreglo para completar } \\
\text { los requisitos del programa sin } \\
\text { sentirme excesivamente estresado. }\end{array}$ & 56 & 1 & 4 & 2,46 & ,660 \\
\hline $\begin{array}{l}\text { 29. La cantidad de trabajo que se } \\
\text { nos pide que hagamos es bastante } \\
\text { razonable. }\end{array}$ & 56 & 1 & 4 & 2,57 & ,806 \\
\hline $\begin{array}{l}\text { 30. Tengo un fuerte sentido de } \\
\text { pertenencia a mi grupo de clase. }\end{array}$ & 56 & 1 & 4 & 2,84 &, 757 \\
\hline $\begin{array}{l}\text { 31. Con frecuencia trabajo con los } \\
\text { compañeros en mis clases. }\end{array}$ & 56 & 1 & 4 & 3,05 & ,699 \\
\hline $\begin{array}{l}\text { 32. He discutido frecuentemente } \\
\text { ideas de la asignatura con otros } \\
\text { estudiantes fuera de clase. }\end{array}$ & 56 & 1 & 4 & 2,29 & ,948 \\
\hline $\begin{array}{l}\text { 33. Discutir el material de la } \\
\text { asignatura con otros estudiantes } \\
\text { fuera de las clases me ha ayudado } \\
\text { a lograr una mejor comprensión de } \\
\text { la materia. }\end{array}$ & 56 & 1 & 4 & 2,34 & ,959 \\
\hline $\begin{array}{l}\text { 34. Puedo ver cómo encajan las } \\
\text { materias para hacer un programa } \\
\text { de estudio coherente para mi } \\
\text { especialidad. }\end{array}$ & 56 & 1 & 4 & 2,70 & ,711 \\
\hline $\begin{array}{l}\text { 35. El programa de estudios de mi } \\
\text { especialidad está bien integrado. }\end{array}$ & 56 & 1 & 4 & 2,63 & ,620 \\
\hline N válido (por lista) & 56 & & & & \\
\hline
\end{tabular}

De hecho, si comparamos los resultados de los dos grupos de estudiantes mediante la prueba U de Mann-Whitney, Tabla 11 (prueba específica para comparar dos muestras independientes cuando no se cumplen los supuestos de normalidad), podemos comprobar diferencias significativas en todos los ítems. Más concretamente, estas diferencias significativas se corroboran a un nivel de significancia de 0,01 , en todos los casos excepto en el ítem 32 (discutir las ideas de la asignatura con otros estudiantes fuera de la clase), donde en ambos grupos este era el aspecto menos trabajado. Pero incluso así, en este caso las diferencias serían significativas si adoptamos el nivel de significatividad a 0.05 (el más común en el ámbito de las Ciencias Sociales y Psicología).

En consecuencia, estos datos muestran claramente como la experiencia metodológica de gamificación mediante kahoot desarrollada en el grupo A, desde la perspectiva del propio alumnado, ha permitido desarrollar un entorno de enseñanza-aprendizaje que favorece el aprendizaje y desarrollo de contenidos en el aula, creando un ambiente lúdico, dinámico y motivador.

\subsection{Bloque 3: Kahoot}

Por último, se presentan los resultados obtenidos en los ítems concernientes a la valoración del uso de la herramienta kahoot (Tabla 12 y 13). Estas preguntas sólo se 


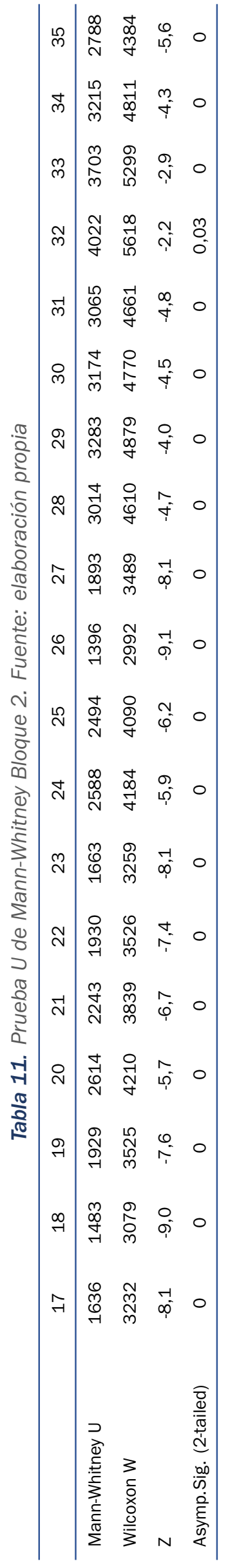


respondieron por el alumnado del grupo A, ya que en el grupo B no se utilizó esta herramienta.

Tabla 12. Valoración herramienta kahoot. Fuente: elaboración propia

\begin{tabular}{lccccc}
\hline & $N$ & Mínimo & Máximo & Media & $\begin{array}{c}\text { Desviación } \\
\text { estándar }\end{array}$ \\
\hline $\begin{array}{l}\text { 36. Considero que la plataforma } \\
\text { Kahoot es adecuada para mejorar } \\
\text { los aprendizajes en el nivel } \\
\text { universitario. }\end{array}$ & 177 & 1 & 4 & 3,57 &, 609 \\
$\begin{array}{l}\text { 37. Después de contestar las } \\
\text { preguntas formuladas en la } \\
\text { plataforma Kahoot, he logrado un } \\
\text { mayor manejo de los } \\
\text { conocimientos y contenidos de la } \\
\text { asignatura. }\end{array}$ & 176 & 2 & 4 & 3,48 &, 623 \\
$\begin{array}{l}\text { 38. Estimo que la herramienta } \\
\text { Kahoot es un recurso que permite } \\
\text { ir mejor preparado para el } \\
\text { momento de realizar el examen }\end{array}$ & 175 & & & & \\
$\mathrm{~N}$ válido (por lista) & & & & & \\
\hline
\end{tabular}

Si tenemos en cuenta los datos que aparecen en la tabla anterior, Tabla 12, se observa que los estudiantes que utilizaron la plataforma Kahoot han valorado su experiencia de manera muy positiva, con valoraciones que oscilan de media entre 3,48 y 3,62 (sobre 4 como valor máximo).

Tabla 13. Descriptivos uso herramienta kahoot. Fuente: elaboración propia

\begin{tabular}{llccc}
\hline & 36 & 37 & 38 \\
\hline $\mathrm{N}$ & Válido & 177 & 176 & 175 \\
& Perdidos & 0 & 1 & 2 \\
Media & & 3,57 & 3,48 & 3,62 \\
Mediana & 4,00 & 4,00 & 4,00 \\
Moda & 4 & 4 & 4 \\
\hline
\end{tabular}

En coherencia, la moda y la mediana de las valoraciones realizadas por los estudiantes se enmarca en el valor 4 (muy de acuerdo). Estos alumnos confirman que el uso de la plataforma Kahoot es adecuado para mejorar los aprendizajes en el nivel universitario, que a través de esta herramienta han logrado un mayor manejo de los conocimientos y contenidos de la asignatura y que han ido mejor preparados para el momento de realizar el examen.

\section{DISCUSIÓN Y CONCLUSIONES}

Se corroboran las finalidades establecidas para esta investigación y el objetivo marcado para este estudio.

En relación con los grandes bloques que componen el cuestionario, se corrobora que el alumnado valora de manera más positiva la creación de entornos de E-A a través de esta experiencia, que el desarrollo de sus capacidades intelectuales, pero en ambos casos las valoraciones son mucho más positivas entre el alumnado que ha desarrollado la experiencia de gamificación que en la muestra del grupo B.

En este sentido, teniendo en cuenta las finalidades que establecíamos para este estudio, podemos confirmar que el alumnado considera que, tras la experiencia, tienen 
una mayor iniciativa, confianza en ellos mismos para seguir aprendiendo, son capaces de resolver problemas y son más efectivos para el grupo de trabajo.

Además, consideran que tras esta vivencia han mejorado la comunicación con el docente, han recibido retroalimentación por parte del profesor y, consideran, que éste se ha esforzado para ellos comprendan los materiales, haciendo especial énfasis en aquellos contenidos que suponían una mayor dificultad para el alumno.

En cuanto al trabajo en equipo, han fomentado el aprendizaje cooperativo, lo que les ha permitido desarrollar las habilidades sociales y personales básicas en un futuro maestro de Educación Primaria. Además, teniendo en cuenta que son alumnos de $1^{\circ}$ curso y $1^{\circ}$ cuatrimestre el trabajo cooperativo ha supuesto un reto para ellos en su proceso de E-A.

Por otra parte, se puede destacar el grado de satisfacción en el grupo A (mañana) por la experiencia de Kahoot, puesto que confirman que favorece su motivación, implicación y diversión, a la vez que aprenden, son capaces de manejar los conocimientos de la asignatura con mayor soltura y, esto les permite ir mejor preparados al examen y obtener mejores resultados.

En definitiva, la experiencia ha resultado altamente gratificante para el alumnado y el profesorado participante en esta experiencia, permitiendo la adquisición de contenidos formativos y habilidades transversales de especial relevancia en la formación de futuros maestros de Educación Primaria en un ambiente educativo retador, estimulante y lúdico. En este sentido, remarcar la importancia de la metodología aplicada en esta experiencia (gamificación) en la formación de estudiantes de magisterios puesto que ha favorecido su implicación, motivación y proceso de aprendizaje. Además, les ha ofrecido la oportunidad de experimentar y conocer una nueva metodología que un futuro podrán utilizar en sus aulas de primaria.

\section{REFERENCIAS}

Amor-Almedina, M. I., y Serrano-Rodríguez, R. (2019). Las competencias generales en la formación inicial del profesorado. Un estudio comparativo entre estudiantes, docentes y graduados de los títulos universitarios de educación. Educación XXI, 22(1), 239-261. https://doi.org/10.5944/educXX1.21341

Bozu, Z., y Arenaga, S. (2017). La formación inicial de maestros y maestras a debate: ¿Qué nos dicen sus protagonistas? Profesorado. Revista de currículum y formación del profesorado, 21(1), 143-163. Descargado de https://recyt.fecyt.es/index.php/ profesorado/article/view/58056

Cerro, G. D. (2015). Aprender jugando, resolviendo: diseñando experiencias positivas de aprendizaje. XII Jornadas Internacionales de Innovación Universitaria Educar para transformar: Aprendizaje experiencial, 20-21 de julio de 2015, UEM.

Contreras, R. S., y Eguía, J. L. (2016). Gamificación en aulas universitarias (Vol. 10). Bellaterra: Institut de la Comunicació (UAB).

Fernández-March, A. (2006). Metodologías activas para la formación de competencias. Educatio Siglo XXI, 24, 35-56. Descargado de https://revistas.um.es/educatio/article/ view/152

Fuertes, A., García, M., Castaño, M. A., López, E., Zacares, M., Cobos, M., ... Grimaldo, F. (2016). Uso de herramientas de respuesta de audiencia en la docencia presencial universitaria. Un primer contacto. Fornadas de Enseñanza Universitaria de la Informática (fENUI) (pp. 261-268). Descargado de http://hdl.handle.net/2117/90357

Gairín-Sallán, J., Díaz-Vicario, A., del Arco Bravo, I., y Alarcia, O. F. (2019). Efecto e impacto de las prácticas curriculares de los Grados de Educación Infantil y Primaria: la perspectiva de estudiantes, tutores y coordinadores. Educación XXI, 22(2), 17-43. https://doi.org/10.5944/educXX1.21311 
García, L. M., y Álvarez Méndez, J. M. (2005). La formación del profesorado universitario para la innovación en el marco de la integración del Espacio Europeo de Educación Superior. Revista de Educación, 337, 51-70. Descargado de http://www.educacionyfp .gob.es/dam/jcr:4555bf2c-d384-4dea-89a7-25b276d802cc/re33704-pdf

Gewerc, A., y Alonso-Ferreiro, A. (2017). Influencias del plan de estudios del grado de maestro de primaria en el conocimiento profesional del profesorado. El caso de la Universidad de Santiago de Compostela. Profesorado. Revista de Currículum y Formación del Profesorado, 21(1), 39-58. Descargado de https://recyt.fecyt.es/index.php/ profesorado/article/view/58047/35563

Guzmán-Duque, A., Mendoza-Paredes, J., y Tavera-Castillo, N. (2018). Kahoot!: un mecanismo de innovación para la educación universitaria. En R. Roig-Vila (Ed.), El compromiso académico y social a través de la investigación e innovación educativas en la Enseñanza Superior (pp. 633-640). Barcelona: Octaedro.

Higgins, E. T., Grant, H., y Shah, J. (1999). Self Regulation and quality of life: Emotional and nonemotional life experiences. En D. Kahneman, E. Diener, y N. Schwarz (Eds.), Wellbeing: The foundations of hedonic psychology (pp. 244-266). Nueva York: Russell Sage Foundation.

Kember, D., y Leung, D. (2009). Development of a questionnaire for assessing students' perceptions of the teaching and learning environment and its use in quality assurance. Learning Environments Research(12), 15-29. https://doi.org/10.1007/s10984-008-9050-7

Kenny, R., y McDaniel, R. (2011). The role teachers' expectations and value assessments of video games play in their adopting and integrating them into their classrooms. British Journal of Educational Technology, 42(2), 197-213. https://doi.org/10.1111/j.1467-8535 2009.01007.x

Kirriemuir, J., y Mcfarlane, A. (2004). Literature Review in Games and Learning. Descargado de https://telearn.archives-ouvertes.fr/hal-00190453/document

López-Belmonte, J., Segura-Robles, A., Fuentes-Cabrera, A., y Parra-González, M. E. (2020). Evaluating Activation and Absence of Negative Effect: Gamification and Escape Rooms for Learning. International fournal of Environmental Research Public Health, 17(7), 2224. https://doi.org/10.3390/ijerph17072224

Medina-Moya, J. L., y Pérez-Cabrera, M. J. (2017). La construcción del conocimiento en el proceso de aprender a ser profesor: la visión de los protagonistas. Profesorado. Revista de Currículum y Formación del Profesorado, 21(1), 17-38. Descargado de https://recyt .fecyt.es/index.php/profesorado/article/view/58042

Mitchell, A., y Savill-Smith, C. (2004). The use of computer and video games for learningA review of the literature. London: Learning and Skills Development Agency.

Montes-Rodríguez, A. J. (2018). La gamificación como metodología didáctica: Una experiencia real en el aula. Independently Published.

Moya, M. M., Carrasco, M., Jiménez, M. A., Ramón, A., Soler, C., y Vaello, M. T. (2016). El aprendizaje basado en juegos: experiencias docentes en la aplicación de la plataforma virtual “Kahoot”. En M. T. Tortosa-Ibáñez, S. Grau-Company, y J. D. Álvarez Teruel (Eds.), XIV Jornadas de Redes de Investigación en Docencia Universitaria. Investigación, innovación y enseñanza universitaria: enfoques pluridisciplinares (pp. 1241-1254). Alicante: Universitat d'Alacant-Institut de Ciències de l'Educació.

Oliva, H. (2017). La gamificación como estrategia metodológica en el contexto educativo universitario. Realidad y Reflexión, 44(0), 29-47. Descargado de http://icti.ufg.edu.sv/ doc/RyRN44-nOliva.pdf

Parra-González, M. E., y Segura-Robles, A. (2019). Traducción y validación de la escala de evaluación de experiencias gamificadas (GAMEX). Bordón. Revista De Pedagogía, 7(4), 87-99. https://doi.org/10.13042/Bordon.2019.70783

Perrotta, C., Featherstone, G., Aston, H., y Houghton, E. (2013). Game-based learning: latest evidence and future directions (NFER Research Programme: Innovation in Education). Slough: NFER. Descargado de https://www.nfer.ac.uk/publications/game01/game01 
.pdf

Pintor-Díaz, P. (2017). Gamificando con Kahoot en Evaluación Formativa. Educación y Aprendizaje (IEYA), 3(2), 112-117. https://doi.org/10.22370/ieya.2017.3.2.709

Sampieri, R. H., Collado, C. F., y Lucio, P. B. (2014). Metodología de la investigación. México: Mcgraw-Hill.

Zabalza, M. A. (2007). Competencias docentes del profesorado universitario: calidad del desarrollo profesional. Madrid: Narcea. 